Anfälleu gelitten haben. Derartige Patienten, ebenso Alkoboliker, soll man nur ganz vorsichtig mit kleineren Dosen behandeln.

3. Richter. Welche Bedeutung hat der "mal franzoso" in Italien in der ersten Hälfte des 15. Jahrhunderts für die Ansicht vom amerikanischen Ursprung der Syphilis. pag. 34 .

Richter ist im Gegensatz zu Sudhoff der Ansicht, daß die Lues aus Amerika eingeschleppt wurde. Daf bei der "Belagerung" von Neapel die erste größere Syphilisepidemie aufgetreten sein soll, ist eine Legende. Das Nähere über die interessante Arbeit ist im Original nachzulesen.

Edgar Braend le (Breslau).

\title{
Dermatologische Wochenschrift,
}

\author{
Bd. LV. Nr. 51a.
}

Goldberg, Lydia. Üb er die Entstehung von Herzklappenfehlern und Aortenaneurysmen durch Syphilis. pag. 1539 .

An der Züricher medizinischen Klinik wurden 37 Fälle von Herzfehlern serologisch untersucht. Von den zwei reinen Aorteninsuffizienzen, bei denen die serologische Untersuchung negativ ausgefallen, hatte die eine Gelenksrheumatismus als Ursache, ebenso 3 von den 4 ebenso reagierenden Fällen von mit Insuffizienz und Stenose der Mitralis kombinierten Aorteninsuffizienzen. Von den 37 Fällen kamen 14 ad exitum und bei 7 lautete die pathologisch-anatomische Diagnose auf Mesaortitis. Bei den übrigen sezierten Fällen waren keine Zeichen überstandener Lues festzustellen. 29 Fälle $=78.4 \%$ waren Männer, $8=21.6 \%$ Weiber. Die unterste Altersgrenze war das 22., die oberste das 66. Lebensjahr. Übereinstimmend mit den serologischen Untersuchungsresultaten standen inbezug auf luetische Átiologie obenan die Aneurysmen der Aorta, dann die mit Tabes kombinierten Klappenfehler und die reinen Aorteninsuffizienzen. Bei reinen Mitralfehlern ist diese Ätiologie wenig nennenswert.

Bd. LVI. Nr. 1.

Unna, P. H. und Golodetz, L. Über Granopla sma und eine allgemeine Methode zur mikrochemischenErforschung eiweißartigerZellbestandteile. pag. 1 .

Interessante Untersuchungen über die chemische Natur des Granoplasma, bezüglich deren Details auf das Original ver- 
wiesen sei. Die Lösungsverhältnisse allgemeinen Charakters zeigen, daß es sich beim Granoplasma um eine Albumose handelt u. zw. um eine aus Akroalburnose hervorgegangene Deuteralbumose, die sich aus den Zellen extrahieren läßt (Zytose), und die durch ihre Reaktion, ihre Färbeverhältnisse charakterisiert ist. Spezifische Lösungsmittel des Granoplasma sind Borsäure, Ferrozyankalium, Chlorbaryum, Plumbum aceticum. Die so gewonnene makrochemische Substanz kann man, wenn sie durch die Extraktion denaturiert ist, durch Einbettung in Celoidin wieder unter mikrochemische Verhältnisse zurückbringen und den spezifischen Färbungen auf Granoplasma unterwerfen, oder man muß die nicht denaturierte Eiweißlösung in kapillare Hohlräume organischer Körper aufsangen (z. B. in Hollundermalz) und sie dann denselben Lösungsmitteln aussetzen.

Fontana, A. Über die Sensibilität der spitzen Kondylome. pag. 17 .

Aus den Untersuchungen Fontanas geht hervor, daß die spitzen Kondylome, wenn auch in geringerem Maße als die normale Haut, mit Tast-, Wärme- und Schmerzempfindlichkeit versehen sind. Infolgedessen kann die Anwesenheit von Nerven und Nervenendigungen in ihnen nicht angezweifelt werden, obzwar die histologischen Untersuchungen mancher Autoren Nervenendigungen daselbst nicht nachweisen konnten.

$$
\text { Nr. } 2 .
$$

AImkvist, J. Über die Bedeutung des Salvarsan und Neosalvarsan bei der Behandlung derSyphilis. pag. 41 .

Die Kombination von Salvarsan (resp. Neosalvarsan) mit $\mathrm{Hg}$ ist die beste Behandlung gegen Lues. 1. Abortivbehandlung: a) 8 intravenöse Injektionen à $0 \cdot 4-0 \cdot 6$ jeden $4 .-5$. Tag, also $3 \cdot 2-4 \cdot 0 \mathrm{~g}$ Salvarsan (4.0-7.3 Neosalvarsan), b) Kuren mit kräftigem Beginn der Behandlung und etwas schwächerer während einer längeren Zeit, also zuerst $\mathrm{Hg}$-Kur ( 7 Injektionen à $0 \cdot 1-0.15 \mathrm{~cm}$ Ol. mercurioli $45 \%$ jeden 5 . Tag). Nach einer Pause von 6-8 Wochen eine ähnliche II. Hg-Kur. Während dieser Zeit 8 intravenöse Salvarsaninjektionen à $0 \cdot 4-0 \cdot 6$. Bei bestehendem erstem Exanthem oder + W.-R. gibt A. vor den großen Salvarsandosen schwächere $\left(0^{\circ} 2-0.3\right)$ oder $3-4$ Injektionen mit Hg salicyl. oder Asurol. (à 0.5 jeden 3. Tag.) Die klinische Beobachtung und Kontrolle der W.R. ergibt die Vorzüge der Methode. 1 b) Was Neosalvarsan betrifft, so scheint dessen therapeutische Wirkung mindestens so gut, wahrscheinlich noch besser als die des Salvarsan. Für die Herstellung frischdestillierten Wassers gibt A. einen einfachen 
Apparat an. Unter 259 Fällen sah er 3 Neurorezidive mit + W.-R. (2 Neuritis acustica, 1 Neuritis optica), niemals aber bei den mit Hg und Salvarsan oder Salvarsan allein energisch behandelten Fällen. Zweimal trat leichte Polyneuritis auf.

Scheven, K. Die ethischen Wirkungen der Reglementierung. pag. 22 und 53.

Vortrag, der die bekannten abolitionistischen Anschauungen und Gründe gegen die Reglementierung der Prostitution enthält.

\section{Nr. 3.}

Ehrlich, Martha. Bakteriologis che Untersuchungen bei verschiedenen Haatentzïndungen (Lichtund Kohlensäurereaktionen, Ekzemen, Ulzerationen usw.). pag. 73.

Nicht beendet.

Aumann. Weiteres über die Infektiosität des Blutes Syphilitischer für Kaninchen. pag. 87.

Durch die Kaninchenimpfung mit mikroskopisch spirochaetenfreiem Blut, resp. Blutserum war es Aumann schon früher gelungen, nach 6-8wöchiger Inkubation typische Spirochaeten nachweisen zu können; von 16 Kaninchen, die mit dem spezifischen Material geimpft waren, erwiesen sich 7 als luetisch infiziert. Am besten eignet sich zu diesen Versuchen defibriniertes Blut. Zwischen Blutentnahme und Impfung verstrichen 1-2 Stunden. Die Inkubationszeit ist auch beim Kaninchen eine regelmäßige (6-S Wochen), wenn man nicht erst abwartet, bis sich die Herde im Hoden tasten lassen, sondern das Hodenpunktat untersucht. Der positive Ausfall dieser Untersuchung wurde immer durch die spätere Sektion nach längerer Beobachtungszeit bestätigt.

Nr. 4.

Lenartowicz, J. T. Ein Fall von isolierter primärerGonorrhoe eines akzessorischen Ganges am Penis. pag. 97.

Der gonorrhoisch infizierte akzessorische Gang am Penis $\mathrm{sa} \beta$ an dessen ventraler Seite, $2 \mathrm{~cm}$ hinter dem Sulcus, in Form eines hirsekorngroßen Knötchens. Er war mit einem mehrschichtigen Plattenepithel ausgekleidet und mul demnach unter die ïberzähligen Hautgänge eingereiht werden.

Ehrlich, Martha. Bakteriolog is che Untersuchungen bei verschiedenen Hautentzündungen (Lichtund Kohlensäurereaktionen, Ekzeme, Ulzerationen usw.). pag. 103.

Zusammenfassung: Die Blasen nach Finsen- und Quarzlampenbeleuchtung halten sich während ihres ganzen Be- 
standes steril; die Krusten wurden häufig mit Staphylo- und Streptokokken (selten mit Pseudodiphtheriebazillen) infiziert gefunden (bei den Quarzlampenreaktionen bisher regelmäßig, bei Finsen weniger häufig). Reine Streptokokken wurden nicht, reine Staphylokokkeninfektionen häufig konstatiert. Klinisch waren Unterschiede zwischen den sterilen, den einfach und doppelt infizierten Fällen nicht nachweisbar. (Ähnlich verhielten sich einige Kohlensäureschneereaktionen.) Die starken (auch eitrigen) Kutisreaktionen auf Tuberkulin (Pirquet und Moro) erwiesen sich auffallend steril. Andere medikamentöse Dermatitiden sind teils steril, teils infiziert. Neben der Streptococcen-Impetigo contagiosa wurden wieder einige klinisch atypische Fälle der Impetigo mit reiner Staphylokokkeninfektion gefunden. Es scheint auch eine atypische Impetigo circinata zu geben, die durch Staphylokokken bedingt ist. Beim Ekthyma wurden immer Streptokokken nachgewiesen, oft zusammen mit Staphylokokken. Beim Ekzem fehlten reine Streptokokkeninfektionen; Mischinfektionen mit Staphylo- und Streptokokken waren häufig. In Ulcera cruris waren immer Staphylo-, sehr häufig Streptokokken vorhanden; die letzteren waren mehrmals nur mit der Bouillonmethode nachzuweisen. In vielen Fällen wuchs daneben - meist ohne klinischen Befund Pyozyaneus, über dessen Bedeutung für den Krankheitsprozeß ein bestimmtes Urteil noch nicht abgegeben werden kann.

Ludwig Waelsch (Prag).

\section{Dermatologisches Zentralblatt.}

XVI. Jahrgang. Nr, 2-4.

Hoffmann, K, F. Trichoschisis. Eine bishernicht be obachtete Haarveränderung. Nr. 2. pag. 34 .

Längsspaltung der Haare in der Haarrinde, ohne Knöt. chenbildung noch Auffaserung am Ende, in zwei oder mehrere Äste bei einer Dame, die sich von einer Haarkünstlerin die Haare mechanisch und chemisch hatte ruinieren lassen.

Ullmann, $K$. uber ein neues Prophylaktikum "Vivilakt" gegen venerische Infektion. Nr. 1. pag. 2 und Nr. 2. pag. 38 .

Klinisch an 20 seit dem Gebrauch des Mittels nicht wieder venerisch erkrankten Patienten erprobt, als auch bakteriologisch bezüglich der Einwirkung auf Gonokokken untersucht, stellt sich "Vivilakt", eine Kombination von Wasserstoffsuperoxyd mit Methylpropylphenol, als ein des Versuches wertes Pro- 